\title{
State Estimation for Time-Delay Systems with Markov Jump Parameters and Missing Measurements
}

\author{
Yushun Tan, Qiong Qi, and Jinliang Liu \\ Department of Applied Mathematics, Nanjing University of Finance and Economics, Nanjing, Jiangsu 210023, China \\ Correspondence should be addressed to Jinliang Liu; liujinliang2009@163.com
}

Received 22 January 2014; Accepted 18 February 2014; Published 3 April 2014

Academic Editor: Shuping He

Copyright ( 2014 Yushun Tan et al. This is an open access article distributed under the Creative Commons Attribution License, which permits unrestricted use, distribution, and reproduction in any medium, provided the original work is properly cited.

\begin{abstract}
This paper is concerned with the state estimation problem for a class of time-delay systems with Markovian jump parameters and missing measurements, considering the fact that data missing may occur in the process of transmission and its failure rates are governed by random variables satisfying certain probabilistic distribution. By employing a new Lyapunov function and using the convexity property of the matrix inequality, a sufficient condition for the existence of the desired state estimator for Markovian jump systems with missing measurements can be achieved by solving some linear matrix inequalities, which can be easily facilitated by using the standard numerical software. Furthermore, the gain of state estimator can also be derived based on the known conditions. Finally, a numerical example is exploited to demonstrate the effectiveness of the proposed method.
\end{abstract}

\section{Introduction}

As a class of multimodal systems, Markovian jump systems (MJSs) have received considerable attention in the past two decades [1-6]. The system parameters usually jump in a finite mode set, in which the transitions among different modes are governed by a Markov chain. Due to the fact that many dynamical systems subject to random abrupt variations can be modeled by MJSs, many applications of MJSs can be showed, such as power systems, failure prone manufacturing systems, communication systems, biochemical systems with diverse changes of environmental conditions, and economy system. Quite a number of useful results have been extensively studied, such as stability and stabilization, robust control, optimal control, $H_{\infty}$ control, synchronization, $H_{\infty}$ filtering, and sliding mode control [7-19]. For example, the author in [7] studied the problem of unbiased estimation of Markov jump systems with distributed delays, and sufficient conditions are obtained for the unbiased $H_{\infty}$ filtering scheme to MJSs by stochastic Lyapunov-Krasovskii functional framework. The author in [8] considered robust $H_{\infty}$ control problems for stochastic fuzzy neutral MJSs with parameters uncertainties and multiple time delays, and a sufficient condition and $H_{\infty}$ control criteria are formulated in the form of linear matrix inequalities by selecting appropriate Lyapunov functions. In term of the peak-to-peak filtering problem for a class of MJSs with uncertain parameters, the author in [9] investigated it. Sufficient conditions that the solution of the peak-to-peak filter existed are given by using the constructed Lyapunov functional and linear matrix inequalities. More details on this topic can be found in [20] and the references therein.

In recent years, due to the fact that, for many practical state estimation applications, the problem of state estimation with linear or nonlinear time-delay systems has received much attention, it is of great significance to estimate systems states and then utilize the estimated systems states to achieve certain design objectives. At the same time, in the procedure of state estimator design, time delays cannot be neglected and their existence often results in a poor performance. Some nice results on state estimation for time-delay systems have been showed in the literature [21-23]. Meanwhile, some state estimation problem for JMSs has been hot topics so that many important results have been reported in the literature 
$[16,24,25]$. The author in [16] studied the state estimation and sliding-mode control problems for continuous-time Markovian jump singular systems with unmeasured states. The author in [24] concerned the problem of $H_{\infty}$ estimation for a class of Markov jump linear systems (MJLSs) with timevarying transition probabilities in discrete-time domain. In [25] efficient simulation-based algorithms called particle filters were used to solve the optimal state estimates for a class of jump Markov linear systems. The author in [26] considered state estimation for Markovian jumping delayed continuoustime recurrent neural networks, where only matrix parameters were mode-dependent. Different from the studies [26, 27] studied state estimation problem for a class of discrete-time neural networks with Markovian jumping parameters and mode-dependent mixed time delay, where the discrete and distributed delays were mode-dependent.

Recently, Liu et al. [28] studied the $H_{\infty}$ filter design for Markovian jump systems with time-varying delays. However, these papers do not consider the data missing of sensor in the process of transmission. Motivated by the idea of above papers, we will investigate the problem of state estimation for Markovian jump systems with both time delays and missing measurements. This work is not a simple extension of [28] to MJSs. Our main difficulties come from the state estimator design and missing measurements analysis for the MJSs. Thus, how to design an appropriate state estimator and how to establish a sufficient condition for the existence of the desired state estimator derived are the key problems to be solved. Based on the above analysis, in this paper, we studied state estimator design for MJSs with both missing measurements and time delays via employing a new Lyapunov function and using the convexity property of the matrix inequality. With the proposed method, we established a sufficient condition for the existence of the desired state estimator. Furthermore, the problem of state estimator design is studied; that is, an observer is designed for the MJSs with missing measurements to estimate the states.

In this paper, the problem of state estimator design for MJSs with interval time-varying delay is narrated. A new Lyapunov function is established to obtain less conservative results, in which the lower and upper delay bound of interval time-varying delay is included. Based on above analysis, the item $\int_{t-\tau_{(t)}}^{t} e^{T}(s) Q_{2}\left(\theta_{t}\right) e(s) d s$ can depart into two parts to deal with, respectively, and the convexity of the matrix functions is used to avoid the conservative caused by enlarging $\tau(t)$ to $\tau_{M}$ in the deriving results.

The rest of this paper is organized as follows. Section 2 presents the problem statement and preliminaries. An LMIbased sufficient condition for the existence of the desired state estimator derived is proposed in Section 3. A numerical example is provided in Section 4 and we conclude this paper in Section 5.

$\mathbb{R}^{n}$ and $\mathbb{R}^{n \times m}$ denote the $n$-dimensional Euclidean space and the set of $n \times m$ real matrices; the superscript " $T$ " represents matrix transposition; $\|\cdot\|$ represents the Euclidean vector norm or the induced matrix 2 -norm as appropriate; $I$ is the identity matrix of appropriate dimension. $\mathbb{E}\{x\}$ represents the expectation of $x$ when $x$ is a stochastic variable. $\left[\begin{array}{l}A \\ B\end{array}\right.$ denote a symmetric matrix, where $*$ denotes the entries implied by symmetry, for a matrix $B$ and two symmetric matrices $A$ and $C$. The notation $X>0$ (resp., $X \geq 0$ ), for $X \in \mathbb{R}^{n \times n}$, means that the matrix $X$ is real symmetric positive definite (resp., positive semidefinite).

\section{Problem Statement and Preliminaries}

Fix a probability space $(\Omega, \mathscr{F}$, and $\mathscr{P})$ and consider the following class of uncertain linear stochastic systems with Markovian jump parameters and time-varying delays:

$$
\begin{aligned}
& \dot{x}(t)=A\left(\theta_{t}\right) x(t)+A_{d}\left(\theta_{t}\right) x(t-\tau(t))+A_{\omega}\left(\theta_{t}\right) \omega(t), \\
& y(t)=C\left(\theta_{t}\right) x(t)+C_{d}\left(\theta_{t}\right) x(t-\tau(t))+C_{\omega}\left(\theta_{t}\right) \omega(t), \\
& z(t)=L\left(\theta_{t}\right) x(t)+L_{d}\left(\theta_{t}\right) x(t-\tau(t))+L_{\omega}\left(\theta_{t}\right) \omega(t), \\
& x(t)=\phi(t), \quad \forall t \in\left[-\tau_{M},-\tau_{m}\right] .
\end{aligned}
$$

$x(t) \in \mathbb{R}^{n}$ is the state vector, $y(t) \in \mathbb{R}^{r}$ is the measurement vector, $z(t) \in \mathbb{R}^{p}$ is the signal to be estimated, $\omega(t) \in L_{2}[0, \infty)$ is the exogenous disturbance signal, and $\left\{\theta_{t}\right\}$ is a continuous-time Markovian process which has right continuous trajectories and takes values in a finite set $\mathcal{S}=$ $\{1,2, \ldots, \mathscr{N}\}$ with stationary transition probabilities:

$$
\operatorname{Prob}\left\{\theta_{t+h}=j \mid \theta_{t}=i\right\}= \begin{cases}\pi_{i j} h+o(h), & i \neq j, \\ 1+\pi_{i i} h+o(h), & i=j,\end{cases}
$$

where $h>0, \lim _{h \rightarrow 0}(o(h) / h)=0$, and $\pi_{i j} \geq 0$, for $j \neq i$ is the transition rate from mode $i$ at time $t$ to the mode $j$ at time $t+h$ and

$$
\pi_{i i}=-\sum_{j=1, j \neq i}^{N} \pi_{i j}
$$

In the system (1), the time delay $\tau(t)$ is a time-varying continuous function satisfying the following assumption:

$$
0 \leq \tau_{m} \leq \tau(t) \leq \tau_{M}<\infty, \quad \dot{\tau}(t) \leq \mu, \quad \forall t>0,
$$

where $\tau_{M}$ is the upper bound and $\tau_{m}$ is the lower bound of the communication delay, and $\mu$ is the upper bound of change rate of communication delay.

When considering the data missing in the sensor channel, the actual output of sensor measurements in system (1) can be described as

$$
\begin{aligned}
\tilde{y}(t) & =\Xi y(t) \\
& =\Xi\left[C\left(\theta_{t}\right) x(t)+C_{d}\left(\theta_{t}\right) x(t-\tau(t))+C_{\omega}\left(\theta_{t}\right) \omega(t)\right],
\end{aligned}
$$

where $\Xi=\operatorname{diag}\left\{\xi_{1}, \xi_{2}, \ldots, \xi_{m}\right\}=\sum_{i=1}^{m} \xi_{i} K_{i}, K_{i}=$ $\operatorname{diag}\{\underbrace{0, \ldots, 0}_{i-1}, 1, \underbrace{0, \ldots, 0}_{m-i}\}$, and $\xi_{i}(i=1,2, \ldots, m)$ are unrelated stochastic variables taking values in $[0,1]$. The mathematical expectation and variance of $\xi_{i}$ are $\bar{\xi}_{i}$ and $\sigma_{i}^{2}$, respectively. 
Remark 1. It can be seen from (5) that stochastic $\Xi$ is introduced to reflect the unreliable sensors, which describes the status of the whole sensor and has been extensively studied in the literature such as [29-33]. Generally speaking, different sensor has different failure rate. So it is reasonable to assume that the failure rate for each individual sensor satisfies individual probabilistic distribution, and the elements $\xi_{i}(i=$ $1,2, \ldots, m)$ of the random matrix $\Xi$ correspond to the status of the $i$ th sensor. At one moment, if $\xi_{i}=1$, it indicates that the $i$ th sensor is well working; if $\xi_{i}=0$, it indicates that $i$ th sensor fails completely or data missing in the sensor channel; if $\xi_{i} \in(0,1)$, it means that the $i$ th sensor fails partly. Therefore, while $\Xi=\operatorname{diag}\{1,1, \ldots, 1\}$, it means the status of the whole sensor is in good working condition. Thus the model which we will establish in this paper is more general.

In this paper, considering the data missing of sensor in the process of information communication and based on the measurement $\tilde{y}(t)$, we consider the following state estimator for system (1):

$$
\begin{aligned}
\dot{\hat{x}}(t)= & A\left(\theta_{t}\right) \hat{x}(t)+A_{d}\left(\theta_{t}\right) \widehat{x}(t-\tau(t)) \\
& +G\left(\theta_{t}\right)\left(y_{1}(t)-\widetilde{y}(t)\right), \\
\widehat{y}(t)= & C\left(\theta_{t}\right) \hat{x}(t)+C_{d}\left(\theta_{t}\right) \hat{x}(t-\tau(t)), \\
\widehat{z}(t)= & L\left(\theta_{t}\right) \hat{x}(t)+L_{d}\left(\theta_{t}\right) \hat{x}(t-\tau(t)),
\end{aligned}
$$

where $y_{1}(t)=\Xi \hat{y}(t)=\Xi\left[C\left(\theta_{t}\right) \widehat{x}(t)+C_{d}\left(\theta_{t}\right) \widehat{x}(t-\tau(t))\right]$.

Remark 2. Similar to (5), we also consider the data missing of sensor in the process of information communication for the system (6) of state estimation.

The set $\mathcal{S}$ contains the various operation modes of system (1) and, for each possible value of $\theta_{t}=i, i \in \mathcal{S}$, the matrices connected with "ith mode" will be denoted by

$$
\begin{aligned}
& A_{i}:=A\left(\theta_{t}=i\right), \quad A_{d i}:=A_{d}\left(\theta_{t}=i\right), \\
& A_{\omega i}:=A_{\omega}\left(\theta_{t}=i\right), \\
& C_{i}:=C\left(\theta_{t}=i\right), \quad C_{d i}:=C_{d}\left(\theta_{t}=i\right), \\
& C_{\omega i}:=C_{\omega}\left(\theta_{t}=i\right), \\
& L_{i}:=L\left(\theta_{t}=i\right), \quad L_{d i}:=L_{d}\left(\theta_{t}=i\right), \\
& L_{\omega i}:=L_{\omega}\left(\theta_{t}=i\right),
\end{aligned}
$$

where $A_{i}, A_{d i}, A_{\omega i}, C_{i}, C_{d i}, C_{\omega i}, L_{i}, L_{d i}$, and $L_{\omega i}$ are constant matrices for any $i \in \mathcal{S}$. In this paper we assume that the jumping process $\left\{\theta_{t}\right\}$ is accessible; that is, the operation mode of system (1) is known for every $t \geq 0$.
Set the estimation error $e(t)=\widehat{x}(t)-x(t)$ and $\widetilde{z}(t)=$ $\widehat{z}(t)-z(t)$. Then the following error dynamics of the state estimation system will be showed as follows:

$$
\begin{aligned}
\dot{e}(t)= & \bar{A}_{i} e(t)+\bar{A}_{d i} e(t-\tau(t))+\bar{A}_{\omega i} \omega(t) \\
& +G_{i}(\Xi-\bar{\Xi}) C_{i} e(t)+G_{i}(\Xi-\bar{\Xi}) C_{d i} e(t-\tau(t)) \\
& -G_{i}(\Xi-\bar{\Xi}) C_{\omega i} \omega(t), \\
\widetilde{z}(t)= & L_{i} e(t)+L_{d i} e(t-\tau(t))-L_{\omega i} \omega(t),
\end{aligned}
$$

where

$$
\begin{aligned}
& \bar{A}_{i}=A_{i}+G_{i} \bar{\Xi} C_{i}, \quad \bar{A}_{d i}=A_{d i}+G_{i} \bar{\Xi} C_{d i}, \\
& \bar{A}_{\omega i}=-A_{\omega i}-G_{i} \bar{\Xi} C_{\omega i} .
\end{aligned}
$$

The state estimation problem which is addressed in this paper is to design a state estimator in form of (8) such that

(i) the estimation error system (8) with $\omega(t)=0$ is exponentially stable;

(ii) the $H_{\infty}$ performance $\|\widetilde{z}(t)\|_{2}<\gamma\|\omega\|_{2}$ is sure for all nonzero $\omega(t) \in L_{2}[0, \infty)$ and a prescribed $\gamma>0$ under the condition $e(t)=0$, for all $t \in\left[-\tau_{M},-\tau_{m}\right]$.

Before giving the main results, the following lemmas and definitions are needed in the proof of our main results.

Lemma 3 (see [34]). For any constant matrix $R \in \mathbb{R}, R=$ $R^{T}>0$, vector function $x:\left[-\tau_{M}, 0\right] \rightarrow \mathbb{R}^{n}$, and constant $\tau_{M}>0$ such that the following integration is well defined; then the following inequality holds:

$$
\begin{aligned}
& -\tau_{M} \int_{t-\tau_{M}}^{t} \dot{x}^{T}(s) R \dot{x}(s) d s \\
& \quad \leq\left[\begin{array}{c}
x(t) \\
x\left(t-\tau_{M}\right)
\end{array}\right]^{T}\left[\begin{array}{cc}
-R & R \\
R & -R
\end{array}\right]\left[\begin{array}{c}
x(t) \\
x\left(t-\tau_{M}\right)
\end{array}\right] .
\end{aligned}
$$

Lemma 4 (see [35]). Suppose $\Xi_{1}, \Xi_{2}$, and $\Omega$ are constant matrices of appropriate dimensions, $0 \leq \tau_{m} \leq \tau(t) \leq \tau_{M}$; then

$$
\left(\tau(t)-\tau_{m}\right) \Xi_{1}+\left(\tau_{M}-\tau(t)\right) \Xi_{2}+\Omega<0
$$

if and only if the following two inequalities hold:

$$
\begin{aligned}
& \left(\tau_{M}-\tau_{m}\right) \Xi_{1}+\Omega<0, \\
& \left(\tau_{M}-\tau_{m}\right) \Xi_{2}+\Omega<0 .
\end{aligned}
$$

Definition 5. The system (8) is considered to be exponentially stable in the mean-square sense (EMSS), if there exist constants $\lambda>0, \alpha>0$, such that $t>0$ :

$$
E\left\{\|x(t)\|^{2}\right\} \leq \alpha e^{-\lambda t} \sup _{-\tau_{M}<s<0}\left\{\|\phi(s)\|^{2}\right\} .
$$


Definition 6. For a given function $V: C_{F_{0}}^{b}\left(\left[-\tau_{M}, 0\right], R^{n}\right) \times S \rightarrow$ $R$, its infinitesimal operator $\mathscr{L}[36]$ is defined as

$$
\mathscr{L} V\left(x_{t}\right)=\lim _{\Delta \rightarrow 0^{+}} \frac{1}{\Delta}\left[E\left(V\left(x_{t+\Delta} \mid x_{t}\right)-V\left(x_{t}\right)\right)\right]
$$

\section{Main Results}

Theorem 7. For some given constants $0 \leq \tau_{m} \leq \tau_{M}$ and $\gamma$, the system (8) is exponentially mean-square stable (EMSS) with a prescribed $H_{\infty}$ performance $\gamma$, if there exist $P_{i}>0, Q_{0}>0$, $Q_{1}>0, Q_{2 i}>0, R_{0}>0, R_{1}>0, Z_{1}>0, Z_{2}>0, M_{i k}>0$, and
$N_{i k}>0(i \in \mathcal{S}, k=1,2, \ldots, 5)$ with appropriate dimensions, so that the following matrix inequalities hold:

$$
\Psi=\left[\begin{array}{cccccc}
\Psi_{11} & * & * & * & * & * \\
\Psi_{21} & \Psi_{22} & * & * & * & * \\
\Psi_{31} & \Psi_{32} & \Psi_{33} & * & * & * \\
\Psi_{41}(s) & \Psi_{42}(s) & 0 & -R_{1} & * & * \\
\Psi_{51} & \Psi_{52} & 0 & 0 & \Psi_{55} & * \\
\Psi_{61} & \Psi_{62} & 0 & 0 & 0 & \Psi_{66}
\end{array}\right]<0,
$$

$$
s=1,2,
$$

$$
\sum_{j=1}^{N} \pi_{i j} Q_{2 j} \leq Z_{k}, \quad k=1,2,
$$

where

$$
\begin{aligned}
& \Psi_{11}=P_{i} \bar{A}_{i}+\bar{A}_{i}^{T} P_{i}+Q_{0}+Q_{1}+Q_{2 i}-R_{0}+\tau_{m} Z_{1}+\delta Z_{2}+\sum_{j=1}^{N} \pi_{i j} P_{j} \text {, } \\
& \Psi_{21}=\left[P_{i}^{T} \bar{A}_{d i}-M_{i 1}+N_{i 1}, R_{0}^{T}+M_{i 1},-N_{i 1}, P_{i}^{T} \bar{A}_{\omega i}\right]^{T} \text {, } \\
& \Psi_{22}=\left[\begin{array}{cccc}
-(1-\mu) Q_{2 i}-M_{i 2}-M_{i 2}^{T}+N_{i 2}+N_{i 2}^{T} & * & * & * \\
-M_{i 3}+M_{i 2}^{T}+N_{i 3} & -Q_{0}-R_{0}+M_{i 3}+M_{i 3}^{T} & * & * \\
-M_{i 4}+N_{i 4}-N_{i 2}^{T} & M_{i 4}-N_{i 3}^{T} & -Q_{1}-N_{i 4}-N_{i 4}^{T} & * \\
-M_{i 5}+N_{i 5} & M_{i 5} & -N_{i 5} & -\gamma^{2} I
\end{array}\right], \\
& \Psi_{31}=\left[\begin{array}{c}
\tau_{m} R_{0} \bar{A}_{i} \\
\sqrt{\delta} R_{1} \bar{A}_{i} \\
L_{i}
\end{array}\right], \quad \Psi_{32}=\left[\begin{array}{cccc}
\tau_{m} R_{0} \bar{A}_{d i} & 0 & 0 & \tau_{m} R_{0} \bar{A}_{\omega i} \\
\sqrt{\delta} R_{1} \bar{A}_{d i} & 0 & 0 & \sqrt{\delta} R_{1} \bar{A}_{\omega i} \\
L_{d i} & 0 & 0 & -L_{\omega i}
\end{array}\right], \quad \Psi_{33}=\operatorname{diag}\left\{-R_{0},-R_{1},-I\right\}, \\
& \Psi_{41}(1)=\sqrt{\delta} M_{i 1}^{T}, \quad \Psi_{41}(2)=\sqrt{\delta} N_{i 1}^{T}, \quad \delta=\tau_{M}-\tau_{m} \text {, } \\
& \Psi_{42}(1)=\left[\begin{array}{llll}
\sqrt{\delta} M_{i 2}^{T} & \sqrt{\delta} M_{i 3}^{T} & \sqrt{\delta} M_{i 4}^{T} & \sqrt{\delta} M_{i 5}^{T}
\end{array}\right], \quad \Psi_{42}(2)=\left[\begin{array}{llll}
\sqrt{\delta} N_{i 2}^{T} & \sqrt{\delta} N_{i 3}^{T} & \sqrt{\delta} N_{i 4}^{T} & \sqrt{\delta} N_{i 5}^{T}
\end{array}\right], \\
& \Psi_{51}=\left[\tau_{m} \sigma_{1} C_{i}^{T} K_{1}^{T} G_{i}^{T} R_{0}^{T}, \tau_{m} \sigma_{2} C_{i}^{T} K_{2}^{T} G_{i}^{T} R_{0}^{T}, \ldots, \tau_{m} \sigma_{m} C_{i}^{T} K_{m}^{T} G_{i}^{T} R_{0}^{T}, 0, \ldots, 0\right]^{T} \\
& \Psi_{52}=\left[\Theta_{5 d}, 0,0, \Theta_{5 \omega}\right], \quad \Psi_{55}=\operatorname{diag}\left\{-R_{0},-R_{0}, \ldots,-R_{0}\right\} \text {, } \\
& \Psi_{61}=\left[\sqrt{\delta} \sigma_{1} C_{i}^{T} K_{1}^{T} G_{i}^{T} R_{1}^{T}, \sqrt{\delta} \sigma_{2} C_{i}^{T} K_{2}^{T} G_{i}^{T} R_{1}^{T}, \ldots, \sqrt{\delta} \sigma_{m} C_{i}^{T} K_{m}^{T} G_{i}^{T} R_{1}^{T}, 0, \ldots, 0\right]^{T} \\
& \Psi_{62}=\left[\Theta_{6 d}, 0,0, \Theta_{6 \omega}\right], \quad \Psi_{66}=\operatorname{diag}\left\{-R_{1},-R_{1}, \ldots,-R_{1}\right\} \text {, } \\
& \Theta_{5 d}=\left[0, \tau_{m} \sigma_{1} C_{d i}^{T} K_{1}^{T} G_{i}^{T} R_{0}^{T}, \tau_{m} \sigma_{2} C_{d i}^{T} K_{2}^{T} G_{i}^{T} R_{0}^{T}, \ldots, \tau_{m} \sigma_{m} C_{d i}^{T} K_{m}^{T} G_{i}^{T} R_{0}^{T}, 0, \ldots, 0\right]^{T}, \\
& \Theta_{5 \omega}=\left[0, \ldots, 0,-\tau_{m} \sigma_{1} C_{\omega i}^{T} K_{1}^{T} G_{i}^{T} R_{0}^{T},-\tau_{m} \sigma_{2} C_{\omega i}^{T} K_{2}^{T} G_{i}^{T} R_{0}^{T}, \ldots,-\tau_{m} \sigma_{m} C_{\omega i}^{T} K_{m}^{T} G_{i}^{T} R_{0}^{T}\right]^{T} \text {, } \\
& \Theta_{6 d}=\left[\sqrt{\delta} \sigma_{1} C_{d i}^{T} K_{1}^{T} G_{i}^{T} R_{1}^{T}, \sqrt{\delta} \sigma_{2} C_{d i}^{T} K_{2}^{T} G_{i}^{T} R_{1}^{T}, \ldots, \sqrt{\delta} \sigma_{m} C_{d i}^{T} K_{m}^{T} G_{i}^{T} R_{1}^{T}, 0, \ldots, 0\right]^{T}, \\
& \Theta_{6 \omega}=\left[0, \ldots, 0,-\sqrt{\delta} \sigma_{1} C_{\omega i}^{T} K_{1}^{T} G_{i}^{T} R_{1}^{T},-\sqrt{\delta} \sigma_{2} C_{\omega i}^{T} K_{2}^{T} G_{i}^{T} R_{1}^{T}, \ldots,-\sqrt{\delta} \sigma_{m} C_{\omega i}^{T} K_{m}^{T} G_{i}^{T} R_{1}^{T}\right]^{T}
\end{aligned}
$$

Proof. Introduce a new vector

$$
\zeta^{T}(t)=\left[\begin{array}{lllll}
e^{T}(t) & e^{T}(t-\tau(t)) & e^{T}\left(t-\tau_{m}\right) & e^{T}\left(t-\tau_{M}\right) & \omega^{T}(t)
\end{array}\right]
$$

and two matrices

$$
\begin{aligned}
\Gamma_{1} & =\left[\begin{array}{lllll}
\bar{A}_{i} & \bar{A}_{d i} & 0 & 0 & \bar{A}_{\omega i}
\end{array}\right], \\
\Gamma_{2} & =\left[\begin{array}{lllll}
L_{i} & L_{d i} & 0 & 0 & -L_{\omega i}
\end{array}\right] .
\end{aligned}
$$


The system (8) can be rewritten as

$$
\begin{aligned}
\dot{e}(k)= & \Gamma_{1} \zeta(t)+G_{i}(\Xi-\bar{\Xi}) C_{i} e(t) \\
& +G_{i}(\Xi-\bar{\Xi}) C_{d i} e(t-\tau(t))-G_{i}(\Xi-\bar{\Xi}) C_{\omega i} \omega(t), \\
\tilde{z}(t)= & \Gamma_{2} \zeta(t) .
\end{aligned}
$$

Let $x_{t}(s)=x(t+s),(-\tau(t) \leq s \leq 0)$. Then, the same as [37], $\left\{\left(x_{t}, \theta_{t}\right), t \geq 0\right\}$ is a Markov process. Choose the following Lyapunov functional candidate:

$$
V\left(x_{t}, \theta_{t}\right)=\sum_{i=1}^{4} V_{i}\left(x_{t}, \theta_{t}\right),
$$

where

$$
\begin{aligned}
V_{1}\left(x_{t}, \theta_{t}\right)= & e^{T}(t) P\left(\theta_{t}\right) e(t) \\
V_{2}\left(x_{t}, \theta_{t}\right)= & \int_{t-\tau_{m}}^{t} e^{T}(s) Q_{0} e(s) d s+\int_{t-\tau_{M}}^{t} e^{T}(s) Q_{1} e(s) d s \\
& +\int_{\left.t-\tau_{t} t\right)}^{t} e^{T}(s) Q_{2}\left(\theta_{t}\right) e(s) d s \\
V_{3}\left(x_{t}, \theta_{t}\right)= & \tau_{m} \int_{t-\tau_{m}}^{t} \int_{s}^{t} \dot{e}^{T}(v) R_{0} \dot{e}(v) d v d s \\
& +\int_{t-\tau_{M}}^{t-\tau_{m}} \int_{s}^{t} \dot{e}^{T}(v) R_{1} \dot{e}(v) d v d s \\
V_{4}\left(x_{t}, \theta_{t}\right)= & \int_{t-\tau_{m}}^{t} \int_{s}^{t} e^{T}(v) Z_{1} e(v) d v d s \\
& +\int_{t-\tau_{M}}^{t-\tau_{m}} \int_{s}^{t} e^{T}(v) Z_{2} e(v) d v d s .
\end{aligned}
$$

Let $\mathscr{L}$ be the weak infinite generator of the random process $\left\{x_{t}, \theta_{t}\right\}$. Then, for each $\theta_{t}=i, i \in \mathcal{S}$, taking expectation on it, we obtain

$$
\begin{aligned}
& \mathbb{E}\left\{\mathscr{L} V\left(x_{t}, \theta_{t}\right)\right\} \\
& \leq e^{T}(t)\left(2 P_{i} \bar{A}_{i}\right. \\
& \left.\quad+\sum_{j=1}^{N} \pi_{i j} P_{j}+Q_{0}+Q_{1}+Q_{2 i}+\tau_{m} Z_{1}+\delta Z_{2}\right) e(t) \\
& +2 e^{T}(t) P_{i} \bar{A}_{d i} e(t-\tau(t))+2 e^{T}(t) P_{i} \bar{A}_{\omega i} \omega(t)
\end{aligned}
$$

$$
\begin{aligned}
& -e^{T}\left(t-\tau_{m}\right) Q_{0} e\left(t-\tau_{m}\right)-\int_{t-\tau_{m}}^{t} e^{T}(s) Z_{1} e(s) d s \\
& +\mathbb{E}\left\{\delta \dot{e}^{T}(t) R_{1} \dot{e}(t)\right\}-\tau_{m} \int_{t-\tau_{m}}^{t} \dot{e}^{T}(s) R_{0} \dot{e}(s) d s \\
& -(1-\mu) e^{T}(t-\tau(t)) Q_{2 i} e(t-\tau(t)) \\
& +\int_{\left.t-\tau_{c} t\right)}^{t} e^{T}(s)\left(\sum_{j=1}^{N} \pi_{i j} Q_{2 j}\right) e(s) d s \\
& +\mathbb{E}\left\{\tau_{m}^{2} \dot{e}^{T}(t) R_{0} \dot{e}(t)\right\}-\int_{t-\tau_{M}}^{t-\tau_{m}} \dot{e}^{T}(s) R_{1} \dot{e}(s) d s \\
& -e^{T}\left(t-\tau_{M}\right) Q_{1} e\left(t-\tau_{M}\right)-\int_{t-\tau_{M}}^{t-\tau_{m}} e^{T}(s) Z_{2} e(s) d s .
\end{aligned}
$$

Note that

$$
\begin{gathered}
\int_{t-\tau_{(t)}}^{t} e^{T}(s)\left(\sum_{j=1}^{N} \pi_{i j} Q_{2 j}\right) e(s) d s \\
=\int_{t-\tau_{(t)}}^{t-\tau_{m}} e^{T}(s)\left(\sum_{j=1}^{N} \pi_{i j} Q_{2 j}\right) e(s) d s \\
+\int_{t-\tau_{m}}^{t} e^{T}(s)\left(\sum_{j=1}^{N} \pi_{i j} Q_{2 j}\right) e(s) d s .
\end{gathered}
$$

From (16) and (24), we can derive that

$$
\begin{aligned}
& \int_{\left.t-\tau_{(} t\right)}^{t} e^{T}(s)\left(\sum_{j=1}^{N} \pi_{i j} Q_{2 j}\right) e(s) d s-\int_{t-\tau_{m}}^{t} e^{T}(s) Z_{1} e(s) d s \\
& \quad-\int_{t-\tau_{M}}^{t-\tau_{m}} e^{T}(s) Z_{2} e(s) d s \\
& =\int_{t-\tau_{m}}^{t} e^{T}(s)\left[\sum_{j=1}^{N} \pi_{i j} Q_{2 j}-Z_{1}\right] e(s) d s \\
& \quad+\int_{t-\tau_{(t)}}^{t-\tau_{m}} e^{T}(s)\left(\sum_{j=1}^{N} \pi_{i j} Q_{2 j}\right) e(s) d s \\
& \quad-\int_{t-\tau_{M}}^{t-\tau_{m}} e^{T}(s) Z_{2} e(s) d s \\
& \leq \int_{t-\tau_{m}}^{t} e^{T}(s)\left[\sum_{j=1}^{N} \pi_{i j} Q_{2 j}-Z_{1}\right] e(s) d s \\
& +\int_{t-\tau_{(t)}}^{t-\tau_{m}} e^{T}(s)\left[\sum_{j=1}^{N} \pi_{i j} Q_{2 j}-Z_{2}\right] e(s) d s<0 .
\end{aligned}
$$


It follows from Lemma 3 that

$$
\begin{aligned}
- & \tau_{m} \int_{t-\tau_{m}}^{t} \dot{e}^{T}(s) R_{0} \dot{e}(s) d s \\
\quad \leq & {\left[\begin{array}{c}
e(t) \\
e\left(t-\tau_{m}\right)
\end{array}\right]^{T}\left[\begin{array}{cc}
-R_{0} & R_{0} \\
R_{0} & -R_{0}
\end{array}\right]\left[\begin{array}{c}
e(t) \\
e\left(t-\tau_{m}\right)
\end{array}\right] . }
\end{aligned}
$$

Combining ((23), (25), and (26)) and introducing slack matrices $M_{i}, N_{i}, i=1,2 \ldots 5$, we obtain

$$
\mathbb{E}\left\{\mathscr{L} V\left(x_{t}, \theta_{t}\right)\right\}-\gamma^{2} \omega^{T}(t) \omega(t)+\widetilde{z}^{T}(t) \widetilde{z}(t)
$$

$$
\leq e^{T}(t)\left(2 P_{i} \bar{A}_{i}+\sum_{j=1}^{N} \pi_{i j} P_{j}+Q_{0}+Q_{1}+Q_{2 i}\right.
$$

$$
\left.+\tau_{m} Z_{1}+\delta Z_{2}\right) e(t)
$$$$
+2 e^{T}(t) P_{i} \bar{A}_{d i} e(t-\tau(t))+2 e^{T}(t) P_{i} \bar{A}_{\omega i} \omega(t)
$$

$$
+\left[\begin{array}{c}
e(t) \\
e\left(t-\tau_{m}\right)
\end{array}\right]^{T}\left[\begin{array}{cc}
-R_{0} & R_{0} \\
R_{0} & -R_{0}
\end{array}\right]\left[\begin{array}{c}
e(t) \\
e\left(t-\tau_{m}\right)
\end{array}\right]
$$$$
-e^{T}\left(t-\tau_{M}\right) Q_{1} e\left(t-\tau_{M}\right)
$$$$
-(1-\mu) e^{T}(t-\tau(t)) Q_{2 i} e(t-\tau(t))
$$$$
+\mathbb{E}\left\{\tau_{m}^{2} \dot{e}^{T}(t) R_{0} \dot{e}(t)\right\}-\int_{t-\tau_{M}}^{t-\tau_{m}} \dot{e}^{T}(s) R_{1} \dot{e}(s) d s
$$$$
+\mathbb{E}\left\{\delta \dot{e}^{T}(t) R_{1} \dot{e}(t)\right\}-\gamma^{2} \omega^{T}(t) \omega(t)+\zeta(t)^{T} \Gamma_{2}^{T} \Gamma_{2} \zeta(t)
$$$$
+2 \zeta^{T}(t) M_{i}\left[e\left(t-\tau_{m}\right)-e(t-\tau(t))-\int_{t-\tau(t)}^{t-\tau_{m}} \dot{e}(s) d s\right]
$$$$
-e^{T}\left(t-\tau_{m}\right) Q_{0} e\left(t-\tau_{m}\right)
$$$$
+2 \zeta^{T}(t) N_{i}\left[e(t-\tau(t))-e\left(t-\tau_{M}\right)\right.
$$

$$
\left.-\int_{t-\tau_{M}}^{t-\tau(t)} \dot{e}(s) d s\right]
$$

where $M_{i}^{T}=\left[\begin{array}{lllll}M_{i 1}^{T} & M_{i 2}^{T} & M_{i 3}^{T} & M_{i 4}^{T} & M_{i 5}^{T}\end{array}\right], \quad N_{i}^{T}=$ $\left[\begin{array}{lllll}N_{i 1}^{T} & N_{i 2}^{T} & N_{i 3}^{T} & N_{i 4}^{T} & N_{i 5}^{T}\end{array}\right]$.
Note that

$$
\begin{aligned}
& -2 \zeta^{T}(t) M_{i} \int_{t-\tau(t)}^{t-\tau_{m}} \dot{e}(s) d s \\
& \leq \int_{t-\tau(t)}^{t-\tau_{m}} \dot{e}^{T}(s) R_{1} \dot{e}(s) d s \\
& +\left(\tau(t)-\tau_{m}\right) \zeta^{T}(t) M_{i} R_{1}^{-1} M_{i}^{T} \zeta(t), \\
& -2 \zeta^{T}(t) N_{i} \int_{t-\tau_{M}}^{t-\tau(t)} \dot{e}(s) d s \\
& \leq \int_{t-\tau_{M}}^{t-\tau(t)} \dot{e}^{T}(s) R_{1} \dot{e}(s) d s \\
& +\left(\tau_{M}-\tau(t)\right) \zeta^{T}(t) N_{i} R_{1}^{-1} N_{i}^{T} \zeta(t), \\
& \mathbb{E}\left\{\delta \dot{e}^{T}(t) R_{1} \dot{e}(t)\right\} \\
& =\delta\left[\bar{A}_{i} e(t)+\bar{A}_{d i} e(t-\tau(t))+\bar{A}_{\omega i} \omega(t)\right]^{T} \\
& \times R_{1}\left[\bar{A}_{i} e(t)+\bar{A}_{d i} e(t-\tau(t))+\bar{A}_{\omega i} \omega(t)\right] \\
& +\delta e^{T}(t) C_{i}^{T} \sum_{i=1}^{m} \sigma_{i}^{2} K_{i}^{T} G_{i}^{T} R_{1} G_{i} K_{i} C_{i} e(t) \\
& +\delta e^{T}(t-\tau(t)) C_{d i}^{T} \sum_{i=1}^{m} \sigma_{i}^{2} K_{i}^{T} G_{i}^{T} R_{1} G_{i} K_{i} C_{d i} e(t-\tau(t)) \\
& -\delta \omega^{T}(t) C_{\omega i}^{T} \sum_{i=1}^{m} \sigma_{i}^{2} K_{i}^{T} G_{i}^{T} R_{1} G_{i} K_{i} C_{\omega i} \omega(t),
\end{aligned}
$$

$$
\begin{aligned}
\mathbb{E}\left\{\tau_{m}^{2} \dot{e}^{T}(t) R_{0} \dot{e}(t)\right\} \\
=\tau_{m}^{2}\left[\bar{A}_{i} e(t)+\bar{A}_{d i} e(t-\tau(t))+\bar{A}_{\omega i} \omega(t)\right]^{T} \\
\quad \times R_{0}\left[\bar{A}_{i} e(t)+\bar{A}_{d i} e(t-\tau(t))+\bar{A}_{\omega i} \omega(t)\right] \\
+\tau_{m}^{2} e^{T}(t) C_{i}^{T} \sum_{i=1}^{m} \sigma_{i}^{2} K_{i}^{T} G_{i}^{T} R_{0} G_{i} K_{i} C_{i} e(t) \\
+\tau_{m}^{2} e^{T}(t-\tau(t)) C_{d i}^{T} \sum_{i=1}^{m} \sigma_{i}^{2} K_{i}^{T} G_{i}^{T} R_{0} G_{i} K_{i} C_{d i} e(t-\tau(t)) \\
-\tau_{m}^{2} \omega^{T}(t) C_{\omega i}^{T} \sum_{i=1}^{m} \sigma_{i}^{2} K_{i}^{T} G_{i}^{T} R_{0} G_{i} K_{i} C_{\omega i} \omega(t) .
\end{aligned}
$$

Combining (27)-(29), we can obtain

$$
\mathbb{E}\left\{\mathscr{L} V\left(x_{t}, \theta_{t}\right)\right\}-\gamma^{2} \omega^{T}(t) \omega(t)+\widetilde{z}^{T}(t) \widetilde{z}(t)
$$

$$
\leq e^{T}(t)\left(2 P_{i} \bar{A}_{i}+\sum_{j=1}^{N} \pi_{i j} P_{j}+Q_{0}+Q_{1}+Q_{2 i}+\tau_{m} Z_{1}\right.
$$




$$
\begin{aligned}
& \left.+\delta Z_{2}\right) e(t)+2 e^{T}(t) P_{i} \bar{A}_{d i} e(t-\tau(t)) \\
& +2 e^{T}(t) P_{i} \bar{A}_{\omega i} \omega(t) \\
& +\delta e^{T}(t-\tau(t)) C_{d i}^{T} \sum_{i=1}^{m} \sigma_{i}^{2} K_{i}^{T} G_{i}^{T} R_{1} G_{i} K_{i} C_{d i} e(t-\tau(t)) \\
& -e^{T}\left(t-\tau_{m}\right) Q_{0} e\left(t-\tau_{m}\right)-e^{T}\left(t-\tau_{M}\right) Q_{1} e\left(t-\tau_{M}\right) \\
& -(1-\mu) e^{T}(t-\tau(t)) Q_{2 i} e(t-\tau(t)) \\
& +\delta e^{T}(t) C_{i}^{T} \sum_{i=1}^{m} \sigma_{i}^{2} K_{i}^{T} G_{i}^{T} R_{1} G_{i} K_{i} C_{i} e(t) \\
& +\delta\left[\bar{A}_{i} e(t)+\bar{A}_{d i} e(t-\tau(t))+\bar{A}_{\omega i} \omega(t)\right]^{T} \\
& \times R_{1}\left[\bar{A}_{i} e(t)+\bar{A}_{d i} e(t-\tau(t))+\bar{A}_{\omega i} \omega(t)\right] \\
& -\delta \omega^{T}(t) C_{\omega i}^{T} \sum_{i=1}^{m} \sigma_{i}^{2} K_{i}^{T} G_{i}^{T} R_{1} G_{i} K_{i} C_{\omega i} \omega(t) \\
& +\tau_{m}^{2} e^{T}(t) C_{i}^{T} \sum_{i=1}^{m} \sigma_{i}^{2} K_{i}^{T} G_{i}^{T} R_{0} G_{i} K_{i} C_{i} e(t) \\
& +\tau_{m}^{2} e^{T}(t-\tau(t)) C_{d i}^{T} \sum_{i=1}^{m} \sigma_{i}^{2} K_{i}^{T} G_{i}^{T} R_{0} G_{i} K_{i} C_{d i} e(t-\tau(t)) \\
& -\tau_{m}^{2} \omega^{T}(t) C_{\omega i}^{T} \sum_{i=1}^{m} \sigma_{i}^{2} K_{i}^{T} G_{i}^{T} R_{0} G_{i} K_{i} C_{\omega i} \omega(t) \\
& +\tau_{m}^{2}\left[\bar{A}_{i} e(t)+\bar{A}_{d i} e(t-\tau(t))+\bar{A}_{\omega i} \omega(t)\right]^{T} \\
& \times R_{0}\left[\bar{A}_{i} e(t)+\bar{A}_{d i} e(t-\tau(t))+\bar{A}_{\omega i} \omega(t)\right] \\
& +\left[\begin{array}{c}
e(t) \\
e\left(t-\tau_{m}\right)
\end{array}\right]^{T}\left[\begin{array}{cc}
-R_{0} & R_{0} \\
R_{0} & -R_{0}
\end{array}\right]\left[\begin{array}{c}
e(t) \\
e\left(t-\tau_{m}\right)
\end{array}\right] \\
& -\gamma^{2} \omega^{T}(t) \omega(t)+\zeta(t)^{T} \Gamma_{2}^{T} \Gamma_{2} \zeta(t) \\
& +2 \zeta^{T}(t) M_{i}\left[x\left(t-\tau_{m}\right)-x(t-\tau(t))\right] \\
& +2 \zeta^{T}(t) N_{i}\left[x(t-\tau(t))-x\left(t-\tau_{M}\right)\right] \\
& +\left(\tau(t)-\tau_{m}\right) \zeta^{T}(t) M_{i} R_{1}^{-1} M_{i}^{T} \zeta(t) \\
& +\left(\tau_{M}-\tau(t)\right) \zeta^{T}(t) N_{i} R_{1}^{-1} N_{i}^{T} \zeta(t) .
\end{aligned}
$$

By using Lemma 4 and Schur complement, it is easy to see that (15) and $s=1,2$ are sufficient conditions to guarantee

$$
\mathbb{E}\left\{\mathscr{L} V\left(x_{t}, \theta_{t}\right)\right\}-\gamma^{2} \omega^{T}(t) \omega(t)+\widetilde{z}^{T}(t) \widetilde{z}(t)<0 .
$$

Then, the following inequality can be concluded:

$$
\mathbb{E}\left\{\mathscr{L} V\left(x_{t}, i, t\right)\right\}<-\lambda_{\min }(\Psi) \mathbb{E}\left\{\zeta^{T}(t) \zeta(t)\right\} .
$$

Define a new function as

$$
W\left(x_{t}, i, t\right)=e^{\epsilon t} V\left(x_{t}, i, t\right) .
$$

Its infinitesimal operator $\mathscr{L}$ is given by

$$
\mathscr{W}\left(x_{t}, i, t\right)=\epsilon e^{\epsilon t} V\left(x_{t}, i, t\right)+e^{\epsilon t} \mathscr{L} V\left(x_{t}, i, t\right) .
$$

By the generalized Itô formula [36], we can obtain from (34) that

$$
\begin{aligned}
\mathbb{E}\left\{W\left(x_{t}, i, t\right)\right\}-\mathbb{E}\left\{W\left(x_{0}, i\right)\right\} \\
=\int_{0}^{t} \epsilon e^{\epsilon s} \mathbb{E}\left\{V\left(x_{s}, i\right)\right\} d s+\int_{0}^{t} e^{\epsilon s} \mathbb{E}\left\{\mathscr{L} V\left(x_{s}, i\right)\right\} d s .
\end{aligned}
$$

Then, similar to the method of [1], we can see that there exists a positive number $\alpha$ such that for $t>0$

$$
\mathbb{E}\left\{V\left(x_{t}, i, t\right)\right\} \leq \alpha \sup _{-\tau_{M} \leq s \leq 0}\left\{\|\phi(s)\|^{2}\right\} e^{-\epsilon t} .
$$

Since $V\left(x_{t}, i, t\right) \geq\left\{\lambda_{\min }\left(P_{i}\right)\right\} x^{T}(t) x(t)$, it can be shown from (36) that for $t \geq 0$

$$
\mathbb{E}\left\{x^{T}(t) x(t)\right\} \leq \bar{\alpha}^{-\epsilon t} \sup _{-\tau_{M} \leq s \leq 0}\left\{\|\phi(s)\|^{2}\right\},
$$

where $\bar{\alpha}=\alpha /\left(\lambda_{\min } P_{i}\right)$. Recalling Definition 5, the proof can be completed.

Remark 8. In the above proof, a new Lyapunov function is constructed, and the term $\int_{t-\tau_{(t)}}^{t} x^{T}(s)\left(\sum_{j=1}^{N} \pi_{i j} Q_{2 j}\right) x(s) d s$ in (25) is separated into two parts. It is easy to see that this method is less conservative than the ones in the literature $[5,38]$.

Remark 9. A delay-dependent stochastic stability condition for MJSs with interval time-varying delays is provided in Theorem 7. In the proof of Theorem 7, the convexity property of the matrix inequality is treated in terms of Lemma 4, which need not enlarge $\tau(t)$ to $\tau_{M}$, so the common existed conservatism caused by this kind of enlargement in [3942] can be avoided, and thus the conservative result will be decreased.

Theorem 7 established some analysis results. In the following, the problem of state estimator design is to be considered and the following results can be readily obtained from Theorem 7 .

Theorem 10. For some given constants $\gamma$ and $0 \leq \tau_{m} \leq \tau_{M}$, the augmented system (8) is stochastically stable with a prescribed $H_{\infty}$ performance $\gamma$ if there exist $P_{i}>0, Q_{0}>0, Q_{1}>0$, $Q_{2 i}>0, R_{0}>0, R_{1}>0, Z_{1}>0, Z_{2}>0, \bar{G}_{i}, M_{i k}$, and 
$N_{i k}(i \in \mathcal{S}, k=1,2 \ldots, 5)$ with appropriate dimensions so that the following LMIs hold for a given $\varepsilon>0$ :

$$
\widehat{\Psi}=\left[\begin{array}{cccccc}
\widehat{\Psi}_{11} & * & * & * & * & * \\
\widehat{\Psi}_{21} & \Psi_{22} & * & * & * & * \\
\widehat{\Psi}_{31} & \widehat{\Psi}_{32} & \widehat{\Psi}_{33} & * & * & * \\
\Psi_{41}(s) & \Psi_{42}(s) & 0 & -R_{1} & * & * \\
\widehat{\Psi}_{51} & \widehat{\Psi}_{52} & 0 & 0 & \widehat{\Psi}_{55} & * \\
\widehat{\Psi}_{61} & \widehat{\Psi}_{62} & 0 & 0 & 0 & \widehat{\Psi}_{66}
\end{array}\right]<0,
$$

where

$$
\begin{aligned}
& \widehat{\Psi}_{11}=P_{i} A_{i}+A_{i}^{T} P_{i}+\bar{G}_{i} C_{i}+C_{i}^{T} \bar{G}_{i}^{T}+Q_{0}+Q_{1} \\
& +Q_{2 i}-R_{0}+\tau_{m} Z_{1}+\delta Z_{2}+\sum_{j=1}^{N} \pi_{i j} P_{j} \\
& \widehat{\Psi}_{21}=\left[P_{i}^{T} A_{d i}+\bar{G}_{i} C_{d i}-M_{i 1}+N_{i 1}, R_{0}^{T}+M_{i 1}\right. \text {, } \\
& \left.-N_{i 1},-P_{i}^{T} A_{\omega i}-\bar{G}_{i} C_{\omega i}\right]^{T} \text {, } \\
& \widehat{\Psi}_{31}=\left[\begin{array}{c}
\tau_{m} P_{i} A_{i}+\tau_{m} \bar{G}_{i} C_{\omega i} \\
\sqrt{\delta} P_{i} A_{i}+\sqrt{\delta} \bar{G}_{i} C_{\omega i} \\
L_{i}
\end{array}\right] \\
& \widehat{\Psi}_{32}=\left[\begin{array}{cccc}
\tau_{m} P_{i} A_{d i}+\tau_{m} \bar{G}_{i} C_{d i} & 0 & 0 & -\tau_{m} P_{i} A_{\omega i}-\tau_{m} \bar{G}_{i} C_{\omega i} \\
\sqrt{\delta} P_{i} A_{d i}+\sqrt{\delta} \bar{G}_{i} C_{d i} & 0 & 0 & -\sqrt{\delta} P_{i} A_{\omega i}-\sqrt{\delta} \bar{G}_{i} C_{\omega i} \\
L_{d i} & 0 & 0 & -L_{\omega i}
\end{array}\right], \\
& \widehat{\Psi}_{33}=\operatorname{diag}\left\{-2 \varepsilon P_{i}+\varepsilon^{2} R_{0},-2 \varepsilon P_{i}+\varepsilon^{2} R_{1},-I\right\} \text {, } \\
& \widehat{\Psi}_{51}=\left[\tau_{m} \sigma_{1} C_{i}^{T} K_{1}^{T} \bar{G}_{i}^{T}, \tau_{m} \sigma_{2} C_{i}^{T} K_{2}^{T} \bar{G}_{i}^{T}, \ldots,\right. \\
& \left.\tau_{m} \sigma_{m} C_{i}^{T} K_{m}^{T} \bar{G}_{i}^{T}, 0, \ldots, 0\right]^{T} \\
& \widehat{\Psi}_{52}=\left[\widehat{\Theta}_{5 d}, 0,0, \widehat{\Theta}_{5 \omega}\right] \text {, } \\
& \widehat{\Psi}_{55}=\operatorname{diag}\left\{-2 \varepsilon P_{i}+\varepsilon^{2} R_{0},-2 \varepsilon P_{i}+\varepsilon^{2} R_{0}, \ldots,\right.
\end{aligned}
$$$$
\left.-2 \varepsilon P_{i}+\varepsilon^{2} R_{0}\right\}
$$

$\widehat{\Psi}_{61}=\left[\sqrt{\delta} \sigma_{1} C_{i}^{T} K_{1}^{T} \bar{G}_{i}^{T}, \sqrt{\delta} \sigma_{2} C_{i}^{T} K_{2}^{T} \bar{G}_{i}^{T}, \ldots\right.$,

$$
\begin{aligned}
& \left.\sqrt{\delta} \sigma_{m} C_{i}^{T} K_{m}^{T} \bar{G}_{i}^{T}, 0, \ldots, 0\right]^{T} \\
& \widehat{\Psi}_{62}=\left[\widehat{\Theta}_{6 d}, 0,0, \widehat{\Theta}_{6 \omega}\right], \\
& \widehat{\Psi}_{66}=\operatorname{diag}\left\{-2 \varepsilon P_{i}+\varepsilon^{2} R_{1},-2 \varepsilon P_{i}+\varepsilon^{2} R_{1}, \ldots,-2 \varepsilon P_{i}+\varepsilon^{2} R_{1}\right\} \text {, } \\
& \widehat{\Theta}_{5 d}=\left[0, \tau_{m} \sigma_{1} C_{d i}^{T} K_{1}^{T} \bar{G}_{i}^{T}, \tau_{m} \sigma_{2} C_{d i}^{T} K_{2}^{T} \bar{G}_{i}^{T}, \ldots,\right. \\
& \left.\tau_{m} \sigma_{m} C_{d i}^{T} K_{m}^{T} \bar{G}_{i}^{T}, 0, \ldots, 0\right]^{T}, \\
& \widehat{\Theta}_{5 \omega}=\left[0, \ldots, 0,-\tau_{m} \sigma_{1} C_{\omega i}^{T} K_{1}^{T} \bar{G}_{i}^{T},-\tau_{m} \sigma_{2} C_{\omega i}^{T} K_{2}^{T} \bar{G}_{i}^{T}, \ldots,\right. \\
& \left.-\tau_{m} \sigma_{m} C_{\omega i}^{T} K_{m}^{T} \bar{G}_{i}^{T}\right]^{T}, \\
& \widehat{\Theta}_{6 d}=\left[0, \sqrt{\delta} \sigma_{1} C_{d i}^{T} K_{1}^{T} \bar{G}_{i}^{T}, \sqrt{\delta} \sigma_{2} C_{d i}^{T} K_{2}^{T} \bar{G}_{i}^{T}, \ldots,\right. \\
& \left.\sqrt{\delta} \sigma_{m} C_{d i}^{T} K_{m}^{T} \bar{G}_{i}^{T}, 0, \ldots, 0\right]^{T}, \\
& \widehat{\Theta}_{6 \omega}=\left[0, \ldots, 0,-\sqrt{\delta} \sigma_{1} C_{\omega i}^{T} K_{1}^{T} \bar{G}_{i}^{T},-\sqrt{\delta} \sigma_{2} C_{\omega i}^{T} K_{2}^{T} \bar{G}_{i}^{T}, \ldots,\right. \\
& \left.-\sqrt{\delta} \sigma_{m} C_{\omega i}^{T} K_{m}^{T} \bar{G}_{i}^{T}\right]^{T},
\end{aligned}
$$

and $\Psi_{22}, \Psi_{41}(s), \Psi_{42}(s)$, and $\delta$ are as defined in Theorem 7 .

Moreover, the state estimator gain in the form of (6) is as follows:

$$
G_{i}=P_{i}^{-1} \bar{G}_{i}
$$

Proof. Defining $\bar{G}_{i}=P_{i} G_{i}$, from (15) and using Schur complement, the matrix inequality (15) holds if and only if

$$
\breve{\Psi}=\left[\begin{array}{cccccc}
\widehat{\Psi}_{11} & * & * & * & * & * \\
\widehat{\Psi}_{21} & \Psi_{22} & * & * & * & * \\
\widehat{\Psi}_{31} & \widehat{\Psi}_{32} & \breve{\Psi}_{33} & * & * & * \\
\Psi_{41}^{\prime}(s) & \Psi_{42}(s) & 0 & -R_{1} & * & * \\
\widehat{\Psi}_{51} & \widehat{\Psi}_{52} & 0 & 0 & \widehat{\Psi}_{55} & * \\
\widehat{\Psi}_{61} & \widehat{\Psi}_{62} & 0 & 0 & 0 & \widehat{\Psi}_{66}
\end{array}\right]<0,
$$$$
s=1,2,
$$

where

$$
\breve{\Psi}_{33}=\operatorname{diag}\left\{-P_{i} R_{0}^{-1} P_{i},-P_{i} R_{1}^{-1} P_{i},-I\right\} .
$$

Due to

$$
\left(R_{k}-\varepsilon^{-1} P_{i}\right) R_{k}^{-1}\left(R_{k}-\varepsilon^{-1} P_{i}\right) \geq 0, \quad i \in S, k=0,1,
$$

we can have

$$
-P_{i} R_{k}^{-1} P_{i} \leq-2 \varepsilon P_{i}+\varepsilon^{2} R_{k}, \quad i \in S, k=0,1 .
$$


Substituting $-P_{i} R_{k}^{-1} P_{i}$ with $-2 \varepsilon P_{i}+\varepsilon^{2} R_{k}(k=0,1)$ in (42), we obtain (38), so if (38) holds, we have (15) holds, and from above proof, we know that the desired state estimator gain matrix is $G_{i}=P_{i}^{-1} \bar{G}_{i}$. This completes the proof.

Remark 11. Inequality (45) is used to bound the term $-P_{i} R_{k}^{-1} P_{i}$. This step can be improved by adopting the cone complementary algorithm [43], which is popular in recent control designs. The scaling parameter $\varepsilon>0$ here can be used to improve conservatism in Theorem 10. In addition, Theorem 10 shows that for given $\varepsilon$ we can obtain the state estimator gain by solving a set of LMIs in (38) and (39).

\section{Numerical Example}

In this section, well-studied example is considered to illustrate the effectiveness of above approaches proposed and also to explain the proposed method on state estimator design.

Consider linear Markovian jump systems in the form of (1) with two modes. For modes 1 and 2, the dynamics of system with following parameters [28] are described as

$$
\begin{aligned}
& A_{1}=\left[\begin{array}{ccc}
-3 & 1 & 0 \\
0.3 & -2.5 & 1 \\
-0.1 & 0.3 & -3.8
\end{array}\right], \quad A_{d 1}=\left[\begin{array}{ccc}
-0.2 & 0.1 & 0.6 \\
0.5 & -1 & -0.8 \\
0 & 1 & -2.5
\end{array}\right], \\
& A_{\omega 1}=\left[\begin{array}{l}
1 \\
0 \\
1
\end{array}\right] \text {, } \\
& C_{1}=\left[\begin{array}{lll}
0.8 & 0.3 & 0
\end{array}\right], \quad C_{d 1}=\left[\begin{array}{lll}
0.2 & -0.3 & -0.6
\end{array}\right], \\
& C_{\omega 1}=0.2 \text {, } \\
& L_{1}=\left[\begin{array}{lll}
0.5 & -0.1 & 1
\end{array}\right], \quad L_{d 1}=\left[\begin{array}{lll}
0 & 0 & 0
\end{array}\right], \quad L_{\omega 1}=0 \text {, } \\
& A_{2}=\left[\begin{array}{ccc}
-2.5 & 0.5 & -0.1 \\
0.1 & -3.5 & 0.3 \\
-0.1 & 1 & -2
\end{array}\right], \quad A_{d 2}=\left[\begin{array}{ccc}
0 & -0.3 & 0.6 \\
0.1 & 0.5 & 0 \\
-0.6 & 1 & -0.8
\end{array}\right] \text {, } \\
& A_{\omega 2}=\left[\begin{array}{c}
-0.6 \\
0.5 \\
0
\end{array}\right] \text {, } \\
& C_{2}=\left[\begin{array}{lll}
0.5 & 0.2 & 0.3
\end{array}\right], \quad C_{d 2}=\left[\begin{array}{lll}
0 & -0.6 & 0.2
\end{array}\right], \\
& C_{\omega 2}=0.5 \text {, } \\
& L_{2}=\left[\begin{array}{lll}
0 & 1 & 0.6
\end{array}\right], \quad L_{d 2}=\left[\begin{array}{lll}
0 & 0 & 0
\end{array}\right] . \\
& L_{\omega 2}=0 \text {, }
\end{aligned}
$$

Suppose the initial conditions are given by $x(0)=$ $\left[\begin{array}{lll}0.8 & 0.2 & -0.9\end{array}\right]^{T}, \widehat{x}(0)=\left[\begin{array}{lll}0 & 0.2 & 0\end{array}\right]^{T}$, and the transition probability matrix

$$
\pi=\left[\begin{array}{ll}
0.5 & 0.5 \\
0.3 & 0.7
\end{array}\right]
$$

By Theorem 10, we get the maximum time delay $\tau_{M}=$ 5.9250 for $\tau_{m}=1, \mu=0.5, \varepsilon=10$, and $\gamma=1.2$. Meanwhile,

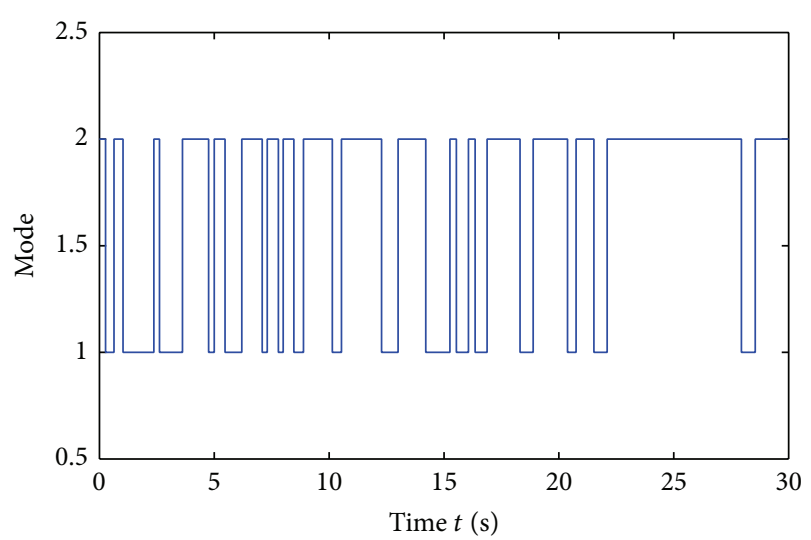

FIgURE 1: Operation modes.

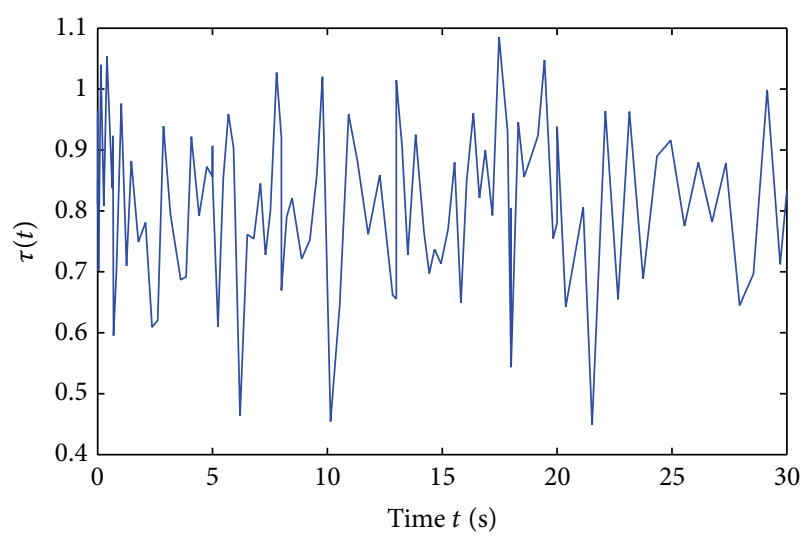

FIGURE 2: Interval time-varying delay.

we can get the fact that the maximum time delay will become larger with decreasing rates of $\tau(t)$ when other variables are fixed. For example, the maximum time delay is $\tau_{M}=6.4072$ for $\mu=0.1$ if other parameters did not change.

The corresponding state estimator gain matrices for $\mu=$ 0.5 are given by

$$
G_{1}=\left[\begin{array}{c}
0.7370 \\
-1.3432 \\
-3.4025
\end{array}\right], \quad G_{2}=\left[\begin{array}{c}
0.7847 \\
0.2051 \\
-1.1228
\end{array}\right] .
$$

To illustrate the performance of the designed state estimator, choose the disturbance function as follows:

$$
\omega(t)= \begin{cases}-0.425, & 5<t<8 \\ 0.375, & 13<t<18 \\ 0, & \text { otherwise }\end{cases}
$$

With this state estimator, the simulation results are shown in Figures 1,2, and 3 which show the operation modes of the MJSs, interval time-varying delay, and estimated signal error $\eta(t)=z(t)-\widetilde{z}(t)$, respectively. From Figures 1, 2, and 3, it can be showed that the designed state estimator performs well. 


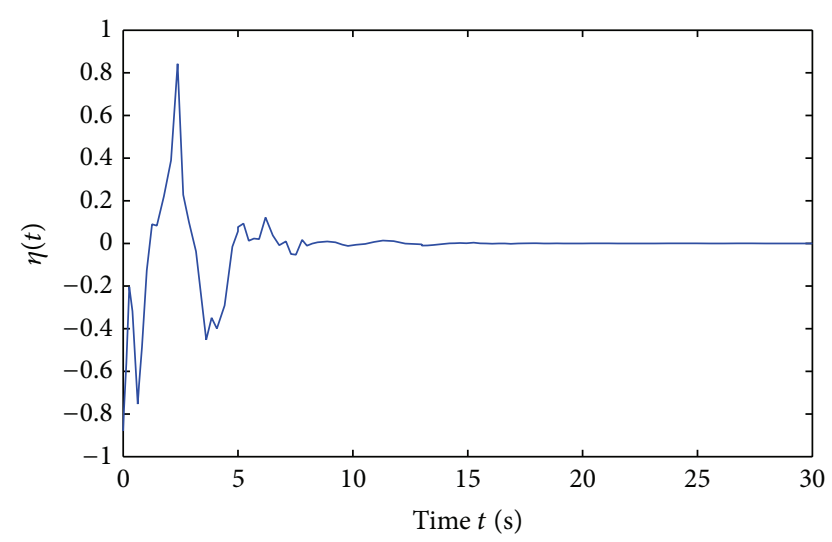

FIGURE 3: Estimated signals error $\eta(t)=z(t)-\widetilde{z}(t)$.

\section{Conclusions}

In this paper, we established the design method of state estimation problem for a class of time-delay systems with Markov jump parameters and missing measurements. By employing a new Lyapunov function method and using the convexity property of the matrix inequality, an LMI-based sufficient condition for the existence of the desired state estimator derived is proposed, which can lead to much less conservative analysis results. Finally, a numerical example has been carried out to show the effectiveness of our obtained results of the proposed method.

\section{Conflict of Interests}

The authors declare that there is no conflict of interests regarding the publication of this paper.

\section{Acknowledgments}

The authors would like to acknowledge the Natural Science Foundation of China (no. 11226240), the Natural Science Foundation of Jiangsu Province of China (no. BK2102469), National Center for International Joint Research on EBusiness Information Processing under Grant 2013B01035, and a project funded by the Priority Academic Program Department of Jiangsu Higher Education Institutions (PAPD).

\section{References}

[1] D. Yue and Q.-L. Han, "Delay-dependent exponential stability of stochastic systems with time-varying delay, nonlinearity, and Markovian switching," IEEE Transactions on Automatic Control, vol. 50, no. 2, pp. 217-222, 2005.

[2] L. Zhang and E.-K. Boukas, "Mode-dependent $H_{\infty}$ filtering for discrete-time Markovian jump linear systems with partly unknown transition probabilities," Automatica, vol. 45, no. 6 , pp. 1462-1467, 2009.

[3] H. Shao, "Delay-range-dependent robust $H^{\infty}$ filtering for uncertain stochastic systems with mode-dependent time delays and Markovian jump parameters," Journal of Mathematical Analysis and Applications, vol. 342, no. 2, pp. 1084-1095, 2008.

[4] O. Costa and M. Fragoso, "A separation principle for the $\mathrm{H}_{2}$ control of continuous-time infinite Markov jump linear systems with partial observations," Journal of Mathematical Analysis and Applications, vol. 331, no. 1, pp. 97-120, 2007.

[5] S. Xu, T. Chen, and J. Lam, "Robust $H^{\infty}$ filtering for uncertain Markovian jump systems with mode-dependent time delays," IEEE Transactions on Automatic Control, vol. 48, no. 5, pp. 900907, 2003.

[6] J. Liu, E. Tian, Z. Gu, and Y. Zhang, "State estimation for Markovian jumping genetic regulatory networks with random delays," Communications in Nonlinear Science and Numerical Simulation, vol. 19, no. 7, pp. 2479-2492, 2014.

[7] S. He and F. Liu, "Unbiased estimation of markov jump systems with distributed delays," Signal Processing, vol. 100, pp. 85-92, 2014.

[8] S. He and F. Liu, "Controlling uncertain fuzzy neutral dynamic systems with Markov jumps," Journal of Systems Engineering and Electronics, vol. 21, no. 3, pp. 476-484, 2010.

[9] S. He and F. Liu, "Robust peak-to-peak filtering for Markov jump systems," Signal Processing, vol. 90, no. 2, pp. 513-522, 2010.

[10] O. L. Costa and W. L. de Paulo, "Indefinite quadratic with linear costs optimal control of Markov jump with multiplicative noise systems," Automatica, vol. 43, no. 4, pp. 587-597, 2007.

[11] H. Zhang, H. Yan, J. Liu, and Q. Chen, "Robust $H_{\infty}$ filters for Markovian jump linear systems under sampled measurements," Journal of Mathematical Analysis and Applications, vol. 356, no. 1, pp. 382-392, 2009.

[12] C. E. de Souza, "Robust stability and stabilization of uncertain discrete-time Markovian jump linear systems," IEEE Transactions on Automatic Control, vol. 51, no. 5, pp. 836-841, 2006.

[13] Z. Wang, Y. Liu, L. Yu, and X. Liu, "Exponential stability of delayed recurrent neural networks with Markovian jumping parameters," Physics Letters A, vol. 356, no. 4-5, pp. 346-352, 2006.

[14] Z. Wang, J. Lam, and X. Liu, "Exponential filtering for uncertain Markovian jump time-delay systems with nonlinear disturbances," IEEE Transactions on Circuits and Systems II, vol. 51, no. 5, pp. 262-268, 2004.

[15] J. Xiong and J. Lam, "Robust $H_{2}$ control of Markovian jump systems with uncertain switching probabilities," International Journal of Systems Science, vol. 40, no. 3, pp. 255-265, 2009.

[16] L. Wu, P. Shi, and H. Gao, "State estimation and slidingmode control of Markovian jump singular systems," IEEE Transactions on Automatic Control, vol. 55, no. 5, pp. 1213-1219, 2010.

[17] L. Hou, G. Zong, W. Zheng, and Y. Wu, "Exponential $l_{2}-$ $l_{\infty}$ control for discrete-time switching markov jump linear systems," Circuits, Systems, and Signal Processing, vol. 32, no. 6, pp. 2745-2759, 2013.

[18] M. G. Todorov and M. D. Fragoso, "A new perspective on the robustness of Markov jump linear systems," Automatica, vol. 49, no. 3, pp. 735-747, 2013.

[19] O. Costa and G. Benites, "Robust mode-independent filtering for discrete-time Markov jump linear systems with multiplicative noises," International Journal of Control, vol. 86, no. 5, pp. 779-793, 2013.

[20] O. L. V. Costa, M. M. D. Fragoso, and R. P. Marques, DiscreteTime Markov Jump Linear Systems, Springer, London, UK, 2005. 
[21] Y. Liu, Z. Wang, J. Liang, and X. Liu, "Synchronization and state estimation for discrete-time complex networks with distributed delays," IEEE Transactions on Systems, Man, and Cybernetics B, vol. 38, no. 5, pp. 1314-1325, 2008.

[22] Z. Wang, Y. Liu, and X. Liu, "State estimation for jumping recurrent neural networks with discrete and distributed delays," Neural Networks, vol. 22, no. 1, pp. 41-48, 2009.

[23] J. Liang, Z. Wang, and X. Liu, "State estimation for coupled uncertain stochastic networks with missing measurements and time-varying delays: the discrete-time case," IEEE Transactions on Neural Networks, vol. 20, no. 5, pp. 781-793, 2009.

[24] L. Zhang, " $H_{\infty}$ estimation for discrete-time piecewise homogeneous Markov jump linear systems," Automatica, vol. 45, no. 11, pp. 2570-2576, 2009.

[25] A. Doucet, N. J. Gordon, and V. Krishnamurthy, "Particle filters for state estimation of jump Markov linear systems," IEEE Transactions on Signal Processing, vol. 49, no. 3, pp. 613-624, 2001.

[26] P. Balasubramaniam, S. Lakshmanan, and S. Jeeva Sathya Theesar, "State estimation for Markovian jumping recurrent neural networks with interval time-varying delays," Nonlinear Dynamics, vol. 60, no. 4, pp. 661-675, 2010.

[27] Y. Liu, Z. Wang, and X. Liu, "State estimation for discretetime Markovian jumping neural networks with mixed modedependent delays," Physics Letters A, vol. 372, no. 48, pp. 7147$7155,2008$.

[28] J. Liu, R. Yan, H. Han, S. Hu, and Y. Hu, " $H_{\infty}$ filtering for Markovian jump systems with time-varying delays," in Proceedings of the IEEE Chinese Control and Decision Conference (CCDC '10), pp. 1249-1254, May 2010.

[29] C. P. Tan and C. Edwards, "Sliding mode observers for robust detection and reconstruction of actuator and sensor faults," International Journal of Robust and Nonlinear Control, vol. 13, no. 5, pp. 443-463, 2003.

[30] E. Tian, D. Yue, and C. Peng, "Brief Paper: reliable control for networked control systems with probabilistic sensors and actuators faults," IET Control Theory and Applications, vol. 4, no. 8, pp. 1478-1488, 2010.

[31] S. Hu, D. Yue, J. Liu, and Z. Du, "Robust $H_{\infty}$ control for networked systems with parameter uncertainties and multiple stochastic sensors and actuators faults," International Journal of Innovative Computing, Information and Control, vol. 8, no. 4, pp. 2693-2704, 2012.

[32] J. Liu, "Event-based reliable h control for networked control system with probabilistic actuator faults," Chinese Journal of Electronics, vol. 22, no. 4, 2013.

[33] J. Liu and D. Yue, "Event-triggering in networked systems with probabilistic sensor and actuator faults," Information Sciences, vol. 240, pp. 145-160, 2013.

[34] K. Gu, V. L. Kharitonov, and J. Chen, Stability of Time-Delay Systems, Birkhäuser Boston, Boston, Mass, USA, 2003.

[35] D. Yue, E. Tian, Y. Zhang, and C. Peng, "Delay-distributiondependent stability and stabilization of T-S fuzzy systems with probabilistic interval delay," IEEE Transactions on Systems, Man, and Cybernetics B, vol. 39, no. 2, pp. 503-516, 2009.

[36] X. Mao, "Exponential stability of stochastic delay interval systems with Markovian switching," IEEE Transactions on Automatic Control, vol. 47, no. 10, pp. 1604-1612, 2002.

[37] E. Boukas, Z. Liu, and G. Liu, "Delay-dependent robust stability and $H_{\infty}$ control of jump linear systems with time-delay," International Journal of Control, vol. 74, no. 4, pp. 329-340, 2001.
[38] S. Xu, J. Lam, and X. Mao, "Delay-dependent $H_{\infty}$ control and filtering for uncertain Markovian jump systems with timevarying delays," IEEE Transactions on Circuits and Systems I, vol. 54, no. 9, pp. 2070-2077, 2007.

[39] B. Chen, X. Liu, and S. Tong, "Delay-dependent stability analysis and control synthesis of fuzzy dynamic systems with time delay," Fuzzy Sets and Systems, vol. 157, no. 16, pp. 2224-2240, 2006.

[40] X. Jiang and Q. Han, "Robust $H_{\infty}$ control for uncertain TakagiSugeno fuzzy systems with interval time-varying delay," IEEE Transactions on Fuzzy Systems, vol. 15, no. 2, pp. 321-331, 2007.

[41] E. Tian and C. Peng, "Delay-dependent stability analysis and synthesis of uncertain T-S fuzzy systems with time-varying delay," Fuzzy Sets and Systems, vol. 157, no. 4, pp. 544-559, 2006.

[42] H. N. Wu and H. X. Li, "New approach to delay-dependent stability analysis and stabilization for continuous-time fuzzy systems with time-varying delay," IEEE Transactions on Fuzzy Systems, vol. 15, no. 3, pp. 482-493, 2007.

[43] L. El Ghaoui, F. Oustry, and M. AitRami, "A cone complementarity linearization algorithm for static output-feedback and related problems," IEEE Transactions on Automatic Control, vol. 42, no. 8, pp. 1171-1176, 1997. 


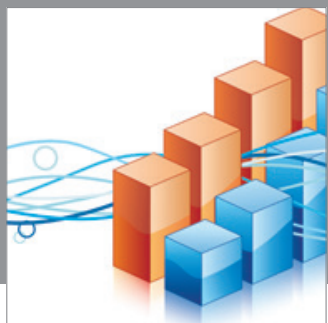

Advances in

Operations Research

mansans

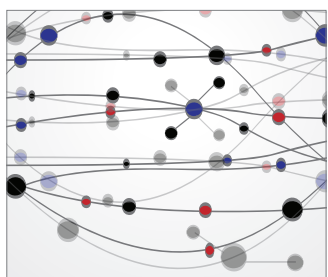

The Scientific World Journal
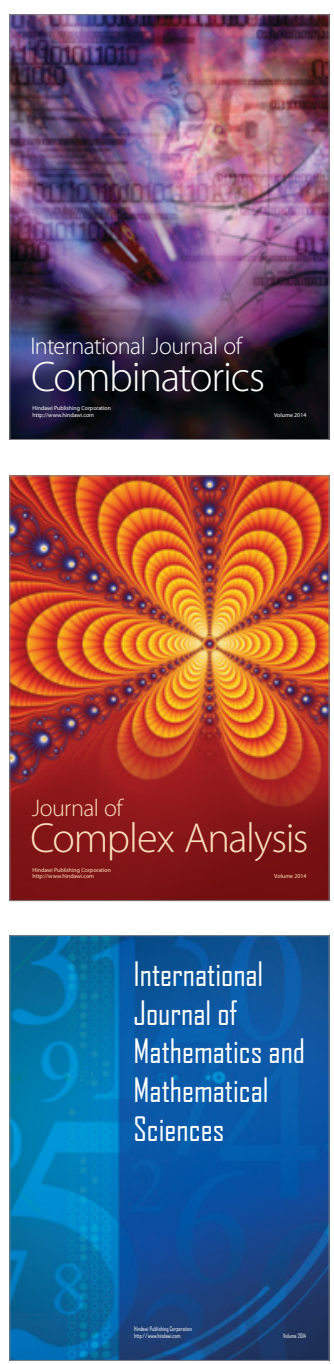
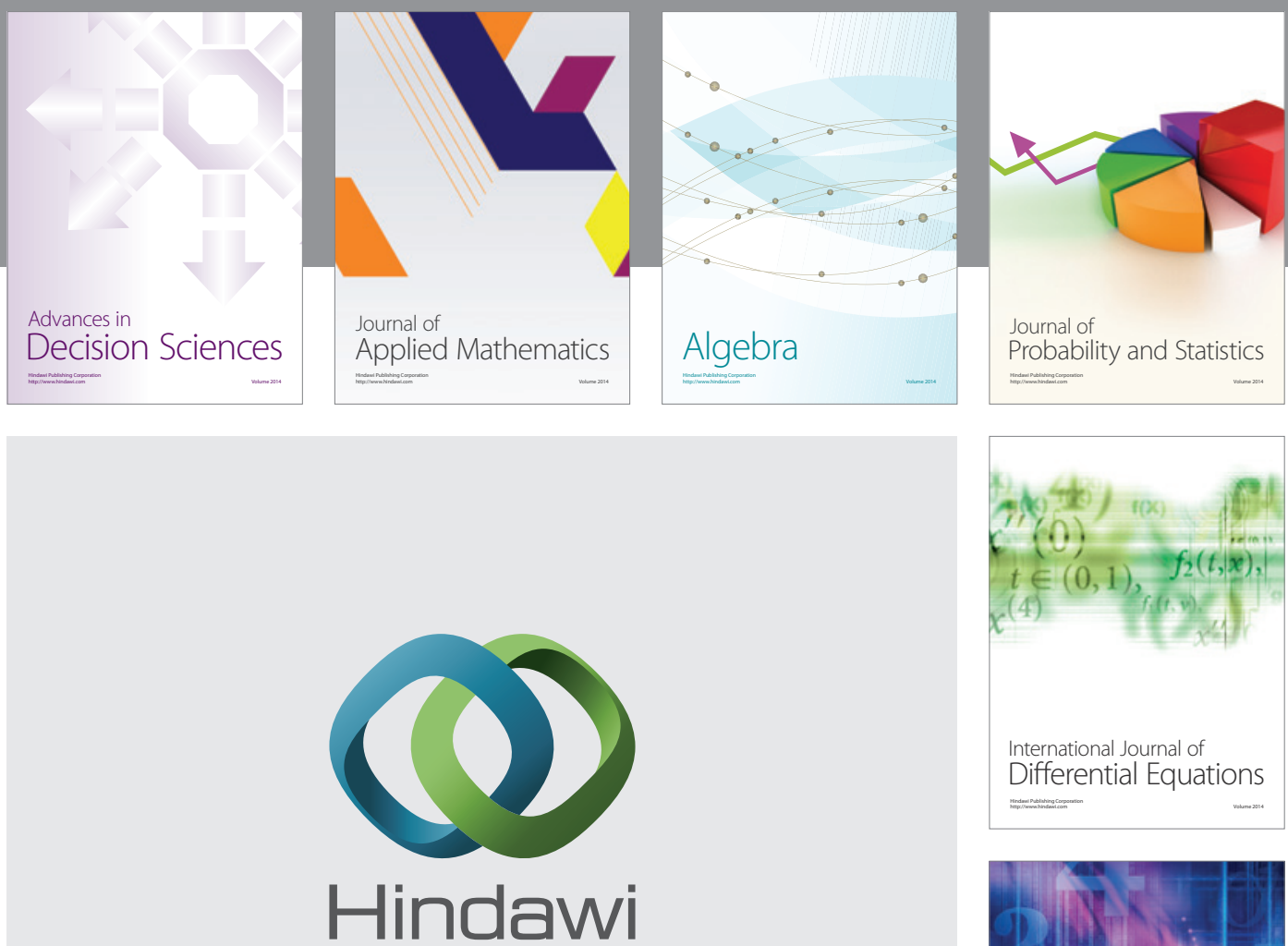

Submit your manuscripts at http://www.hindawi.com
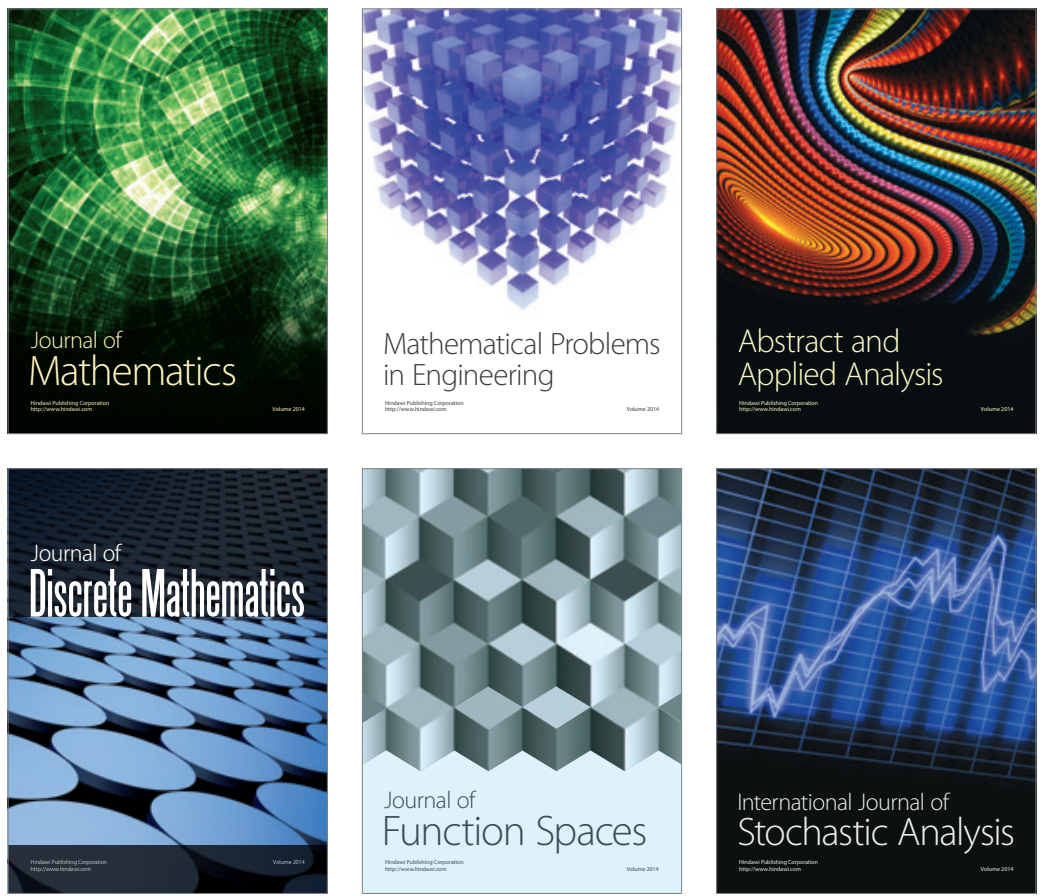

Journal of

Function Spaces

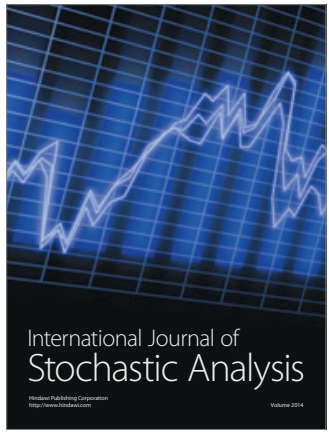

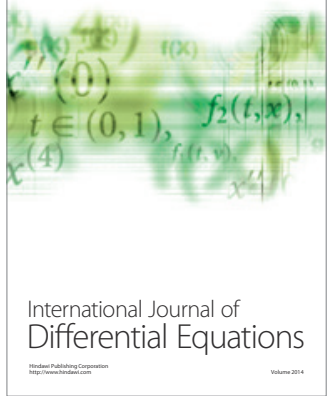
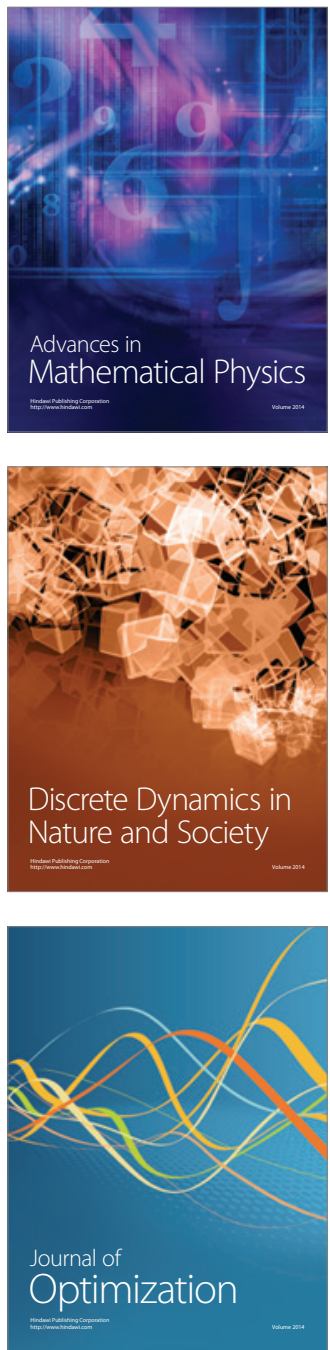